\title{
Marine Vibrator: Source Wavefield Modeling
}

Sander W. Losnedahl*(PGS/UIO), Okwudili C. Orji (PGS), Leiv J. Gelius (UIO), Walter Söllner (PGS)

\section{Summary}

Traditional marine seismic sources are facing increasingly strict regulation due to their non-negligible environmental foot print. However, marine vibrators are easy to control, are more efficient and have lower environmental foot print. Here we demonstrate source wavefield modeling for marine vibrators, discuss the source signature requirements and important processing and imaging steps.

\section{Introduction}

The marine geophysical community proactively seeks to be environmentally responsible. Low environmental footprint in terms of Sound Pressure Level (SPL) and Sound Exposure Level (SEL) especially in locations marked as "sensitive areas" is a major goal for the industry. In addition, though conventional air-gun sources can deliver low frequencies that may be used in some specialized processing (e.g. full waveform inversion), such procedures would work even better if the low frequencies could be further enhanced, which could be achieved using marine vibrators. As a result, several alternatives to conventional air-gun sources have been developed. Tenghamn and Long (2006) present a marine vibrator with a flextensional shell. Dellinger et al. (2016) present a marine vibrator that generates low frequencies. Roy et al. (2018) present a marine vibrator source capable of emitting energy from $10-100 \mathrm{~Hz}$.

One common feature of these sources is that the output pressure is based on the oscillation of their plates.

In this work, we present a method for modeling the output pressure wavefield for a marine vibrator that is based on stacks of oscillating plates. An example of the measured amplitude spectrum of a marine vibrator source which generates acoustic wavefield by actuating a vibrator surface to produce a volume change in the surrounding water is shown in Figure 1 (Oscarsson et al., 2019). The basic element of the source consists of two parallel plates actuated towards and away from each other. When a multitude of these elements are arranged in proximity to each other, they form a modular projector system. The full system is divided into Low Frequency (LF) and High Frequency (HF) modules. The LF module is specially designed to drive a large radiating area at small displacements and was operated from $1-45 \mathrm{~Hz}$ while the HF module was operated from 35 $100 \mathrm{~Hz}$ for $30 \mathrm{~s}$. The equivalence of $5 \mathrm{~s}$ notional was computed based on Rietsch (1977).

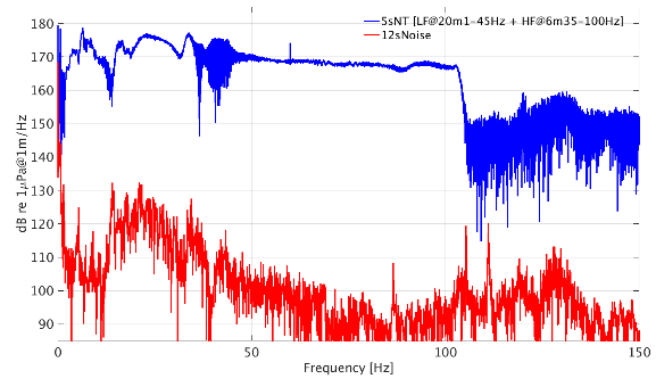

Figure 1: Amplitude spectra of the measured LF and HF sources.

Since marine vibrators are non-impulsive and are in motion during data acquisition, synthetic data was computed and imaged for stationary and moving sources in order to investigate the performance of these types of sources with respect to data acquisition, processing and imaging.

\section{Theory}

The modeling method is based on the reciprocity theorem and relates the acceleration of the plates directly to the output pressure. Hence, the required plate motion can be computed. The building element of the marine vibrator source is composed of an oscillating pair of plates in water, which enclose a volume of air under pressure. The seismic wavefield everywhere outside this volume may be derived from the motion of the vibrator plates, which leads to increasing and decreasing of the enclosed volume (see Figure 2). Inside the plates the acoustic laws may fail and this volume will be removed from the model.

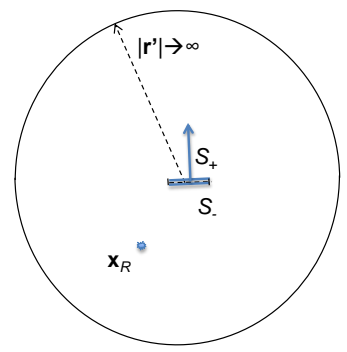

Figure 2: Single vibrator: Plate surface with normal vector across the inner surface

We start from the acoustic representation theorem. The pressure wavefield inside the model enclosed by the spherical surface as outer border and an idealized surface surrounding the oscillating plates, as inner border, is expressed for a model free of body forces by surface integrals of the free space Green's function $g$, the pressure $p$, and the gradients of these wavefields on the outer and 


\section{Source Wavefield Modeling}

inner surfaces (Morse and Feshbach, 1953). By letting the outer surface go to infinity and applying Sommerfeld's radiation condition (Sommerfeld, 1949), the pressure may be written as a surface integral enclosing the plate volume:

$p\left(\boldsymbol{x}_{R}, t\right)=\int_{S_{+}+S_{-}}\left(g\left(\boldsymbol{x}, \boldsymbol{x}_{\boldsymbol{R}}, t\right) * \nabla p(\boldsymbol{x}, t)-\nabla g\left(\boldsymbol{x}, \boldsymbol{x}_{\boldsymbol{R}}, t\right) *\right.$
$p(\boldsymbol{x}, t)) d \boldsymbol{x}$

In equation 1 we have assumed that the surface surrounding the total volume that is removed is given solely by the plate surfaces $S_{+}$and $S_{-}$(i.e., the distance between the plates is much smaller than the plate size). Defining the direction of the normal vector to point from $S_{-}$to $S_{+}$(as indicated in Figure 1), the integral over the entire surface may be expressed as:

$$
\begin{aligned}
& p\left(\boldsymbol{x}_{R}, t\right)=\int_{S_{+}}\left(g\left(\boldsymbol{x}, \boldsymbol{x}_{\boldsymbol{R}}, t\right) * \nabla p(\boldsymbol{x}, t)-\nabla g\left(\boldsymbol{x}, \boldsymbol{x}_{\boldsymbol{R}}, t\right) *\right. \\
& p(\boldsymbol{x}, t)) d \boldsymbol{x}-\int_{S_{-}}\left(g\left(\boldsymbol{x}, \boldsymbol{x}_{\boldsymbol{R}}, t\right) * \nabla p(\boldsymbol{x}, t)-\nabla g\left(\boldsymbol{x}, \boldsymbol{x}_{\boldsymbol{R}}, t\right) *\right. \\
& p(\boldsymbol{x}, t)) d \boldsymbol{x} .
\end{aligned}
$$

So far no assumptions are made about Green's functions or wavefields on the plate surfaces. If we now assume continuity of the pressure fields across the surfaces, which is a valid assumption for thin synchronously oscillating plates separated by a very small distance, and we also impose continuity for the Green's functions and their derivatives we obtain:

$$
p\left(\boldsymbol{x}_{R}, t\right)=\int_{S_{+}} g\left(\boldsymbol{x}, \boldsymbol{x}_{\boldsymbol{R}}, t\right) *[\nabla p(\boldsymbol{x}, t)] d \boldsymbol{x} .
$$

The bracket [.] in equation 3 denotes the difference of values, in this case of the gradients of the pressure wavefield across the plate surfaces. Since an accurate measurement of the pressure gradients at the plate surfaces might be difficult, we replace the pressure gradients by particle accelerations from the equation of motion:

$$
p\left(\boldsymbol{x}_{R}, t\right)=\rho \int_{S_{+}} g\left(\boldsymbol{x}, \boldsymbol{x}_{\boldsymbol{R}}, t\right) *[\boldsymbol{a}(\boldsymbol{x}, t)] d \boldsymbol{x} .
$$

Equation 4 is our final expression for calculating the emitted pressure wavefield from a surface integral of the free space Green's function and the particle acceleration difference across the surfaces of one pair of synchronously oscillating plates. By using the continuity of the normal particle velocity at the plate water contact, the particle acceleration is obtained from the induced plate oscillation (Söllner and Orji, 2018). As a consequence of equation 4 the derived pressure wavefield everywhere in the model will be in phase with the acceleration of the plate oscillation.If we aim for a flat spectral farfield pressure emitted by the source, the time function of the plate motion needs to be designed such that the acceleration becomes a flat function in the frequency domain. If instead plate motion with a flat spectral behavior were generated, this would automatically lead to an emitted pressure wavefield with strongly suppressed low frequencies.

\section{Marine Vibrator Source Wavefield}

Using equation 4, the source wavefield was computed for a pair of circular vibrating plates with $0.5 \mathrm{~m}$ radius. The source wavefield was modeled for $1-20 \mathrm{~Hz} 4 \mathrm{~s}$ long sweeps. The output was computed for a receiver located $100 \mathrm{~m}$ from the center of the oscillating plates. The condition of flat amplitude spectrum of the acceleration was imposed. Figure 3 show the time plot of plate motion (left panel), the plate acceleration (middle panel) and computed pressure (right panel). Figure 4 and 5 show the corresponding amplitude and phase spectra respectively. Observe that, as equation 4 suggests, the amplitude spectrum of the plate motion is proportional to $1 / f^{2}$ since the amplitude spectrum of the plate acceleration is flat (see Figure 4). Note also from Figure 5 (top panel), that the plate motion and the acceleration are 180 degrees out of phase while the pressure and acceleration are in phase (lower panel).
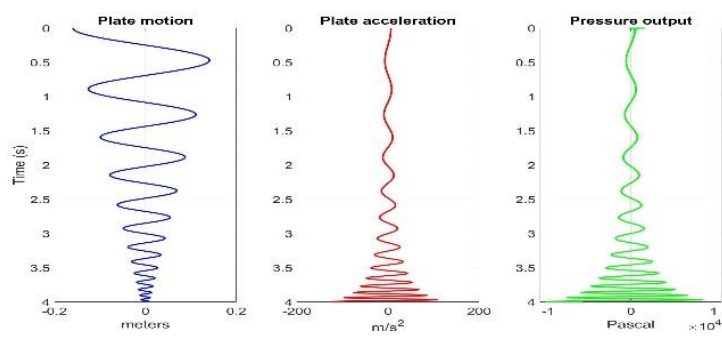

Figure 3: Time plots of the modeled source wavefield.
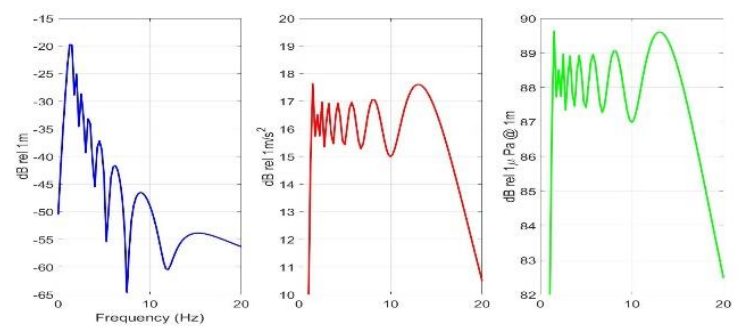

Figure 4: Amplitude spectra of the modeled source wavefield. 


\section{Source Wavefield Modeling}
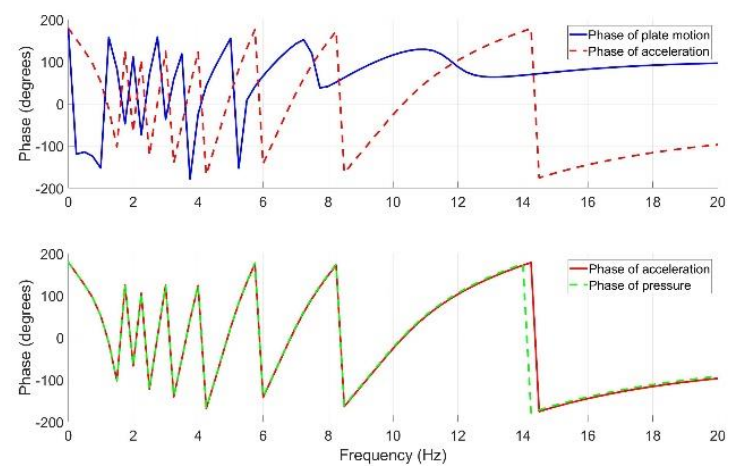

Figure 5: Phase spectra of the modeled source wavefield.

As Figure 4 suggests, much more work is needed to generate the low frequencies in comparison to higher frequencies. Hence, in order to achieve a desired constant pressure output over the entire frequency band, the source could be divided into low frequency (LF) and high frequency (HF) modules (see Figure 1).

\section{Synthetic Data Generation and Imaging}

In order to generate enough output, marine vibrator source signatures are inherently long. Unlike air-gun sources, marine vibrators are moving while generating their wavefields. Hence, the wavefields have motion effects. In order to demonstrate the effect of this motion on imaged targets, synthetic data was generated for stationary and moving sources using on 2D Finite Difference modeling (2DFD).

The data was computed using a homogenous background model consisting of water with two diffractors that are $150 \mathrm{~m}$ apart and located at a depth of $1000 \mathrm{~m}$. Data was computed for a total of 200 shots for 960 receivers that were placed $490 \mathrm{~m}$ away from the sea surface in a split-spread configuration. The source was placed $480 \mathrm{~m}$ from the sea surface and both the shot and receiver intervals were $6.25 \mathrm{~m}$. The modeled source wavelet was a $5-100 \mathrm{~Hz} 5$ s linear sweep. Two data sets were computed for a stationary source and a source moving at $2.67 \mathrm{~m} / \mathrm{s}$ (5.19 knots) while the receivers were stationary. To reduce artefacts from 2DFD modeling, a Perfectly Matched Layers, PML, absorbing boundary was used and the sea surface reflection was turned off.
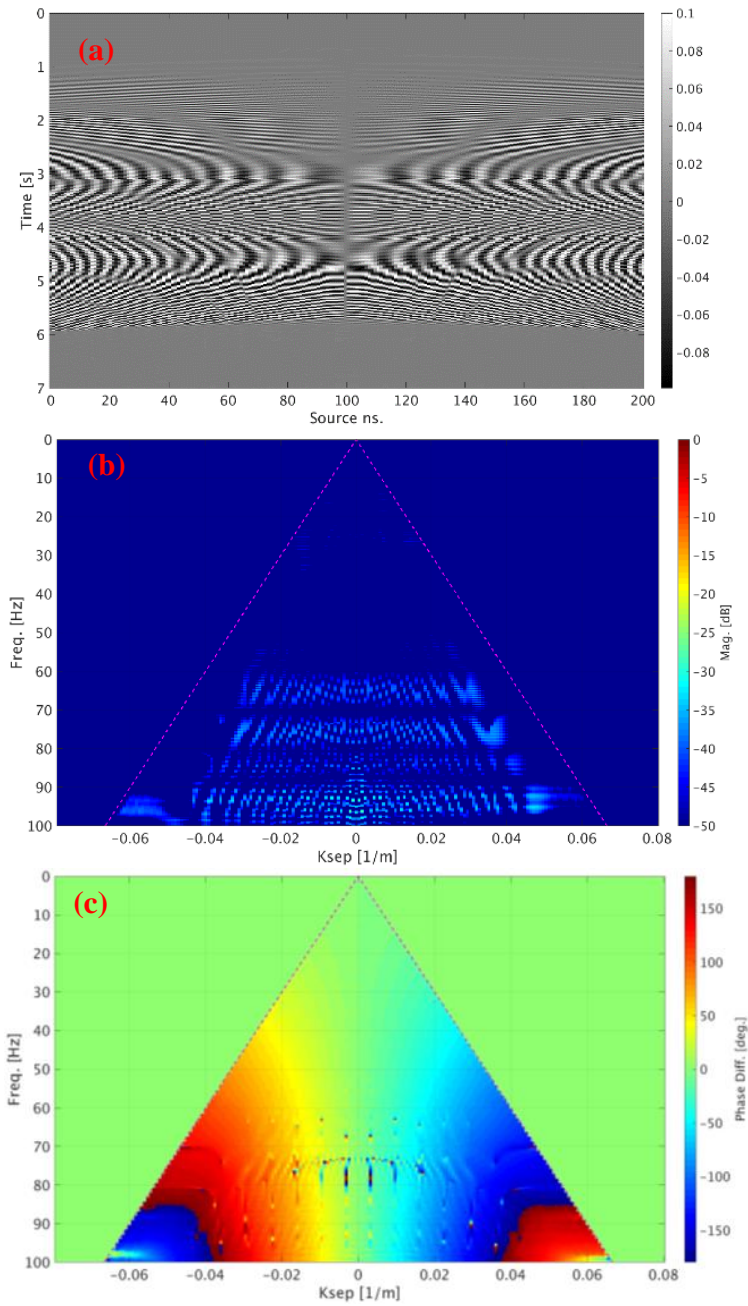

Figure 6: Common receiver gather of moving and stationary datasets scaled by 10 for visualization in TX domain (a), the amplitude ratio in FK-domain (b) and the phase difference in FK-domain (c).

Figure 6a shows a common receiver gather of the difference between the moving and the stationary source data in time and space. The amplitude ratio as shown in Figure $6 \mathrm{~b}$ is negligible (less than about $-35 \mathrm{~dB}$ ). Figure 6c shows the spectrum of the phase difference between moving and stationary sources. The plots show that the problem of a moving source is essentially a phase correction problem. Observe also that since the source is moving from left to right and passing through the top of the diffractors, when the source is located vertically above the diffractors, there is zero net difference in phase and amplitude between the two data sets (see Figure 6a \& 6c) which is as expected according to Doppler effects. Moreover, there is a polarity reversal as the source passes the diffractors' locations (Figure 6a and 


\section{Source Wavefield Modeling}

6c) Figure 6c shows that the phase difference increases with propagation angle which implies that the difference in the time series (Figure 6a) increases with offset. Moreover the difference in amplitude increase from lower time to higher times which is an indication that a linear up sweep was used.

In order to analyse the effect of motion in the final image, the computed data sets were cross-correlated using the pilot trace (that is the modeled source wavefield for a stationary source). This is correct for a stationary source but will introduce errors for the moving source data set (see Figure 6). Subsequently the data sets were migrated using 2D Full Finite Difference Migration, 2DFFDMIG with correlation imaging condition (Schleicher et al., 2008). Figure 7 shows depth slices of the imaged output at the target location for both data sets. The differences obtained from the two images shown in green (see Figure 7) shows that the percentage amplitude difference is at most $3.15 \%$ (or normalized root mean square (NRMS) of $2.85 \%$ NRMS). One may argue that this type of difference may be significant in the context of 4D processing. In such cases, motion could be corrected for by utilizing deconvolution techniques (Hampson and Jakubowicz, 1995; Asgedom et.al., 2019). Nevertheless, the simplified model used in the synthetic data computation and the acquistion configuration may be limitations to a broad interpretation of the results.

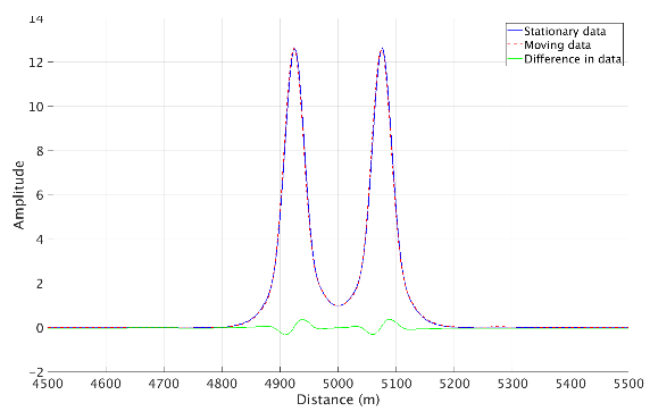

Figure 7: Imaged results for data computed for stationary source (blue), a source moving at speed of $2.67 \mathrm{~m} / \mathrm{s}$ (5.19 knots) (red) and the difference (green).

\section{Discussion and Conclusions}

The theory of reciprocity was used to derive an equation (equation 4) describing a continuous wavefield emitted from a point source. Such a sweep was in this case designed to have a flat amplitude spectrum (Figure 4) which is advantageous for many aspects of seismic data processing. However, the plate motion must behave in a specific manner to sustain such an amplitude spectrum across all frequencies. This causes the phase of the plate motion to be 180 degrees out of phase with the plate acceleration as seen in Figure 5. The linear sweep was used both as a stationary and moving source in finite difference modeling of a simple homogeneous model containing two diffractors. To see the effects of motion on the generated data, the stationary data set was subtracted from the moving data set. The results are shown in Figures 6a-6c. From these figures it can be seen that the amplitude differences are minor, but the phase can vary by up to 180 degrees. This phase change is due to the source moving relative to the diffractors, in contrast to a stationary source. The direction of motion of the source can be inferred from the data - with increasing shot number, the source moves closer to the diffractors and passes directly above them, then moves away from the diffractors' location. This is important as the direction of motion relative to the target determines if the pressure wavefield is compressed or dilated. Compressing the wavefield will generate higher frequencies, while dilation will generate lower frequencies. However, given the very low source speeds in marine seismic acquisition, any shift towards new frequencies by compression and dilation may not be visible as Figure 7 shows.

Significant phase differences were observed between the receiver gather of the stationary and the moving sources. Moreover, the source motion was not taken into account during processing and imaging. However, the net difference between the two migrated data sets is at most $3.15 \%$. Nevertheless, a simplified geological model was used in this study, a further study with a more complex geology is ongoing.

\section{Acknowledgements}

The authors would like to thank PGS for permission to publish this work. A special thanks to the University of Oslo for their support. 